\title{
ÜBERLEGUNGEN ZUR TRANSKULTURELLEN DIMENSION DER MEHRSPRACHIGKEIT AUS SOZIOLINGUISTISCHER SICHT
}

\author{
Magdalena Malechová \\ Jihočeská univerzita v Českých Budějovicích, \\ Filozofická fakulta, Ústav česko-německých areálových studií a germanistiky, \\ Branišovská 31a, 37005 České Budějovice, Česká republika \\ e-mail: malechova@ff.jcu.cz
}

\begin{abstract}
Transkulturalität stellt als gegenwärtiges Phänomen die Begegnung zweier oder mehrerer Kulturen dar, die unterschiedliche oder gegensätzliche Aspekte aufweisen. Die Kultur jedes einzelnen Volkes ist einzigartig, weist einmalige und individuelle Beschaffenheiten und Eigenarten auf. Wenn also zwei bzw. mehrere Kulturen aufeinandertreffen, kommt es zur Begegnung mannigfaltiger Charakteristika der jeweiligen Völker. Die Sprache gehört zu einem der wichtigsten Vermächtnisse, die uns als kulturelles Erbe hinterlassen wurden. Die heutige moderne wirtschaftliche und gesellschaftliche Entwicklung, die man z. B. an Kommunikationsmedien beobachten kann, zwingt jedoch uns Sprachbenutzer, uns den Herausforderungen der neuesten Medien zu stellen und im Rahmen der gegenwärtigen globalisierten Welt unsere Kultur mit den anderen Nationen zu teilen und sie ,über die Grenzen hinaus“ zu präsentieren.
\end{abstract}

\section{Keywords}

Trans-cultural character; Multilingualism; Language context; Translation; Headline.

\section{Einleitung}

Die Begriffe Transkulturalität oder Mehrsprachigkeit, als Fachbegriffe jeweiliger Untersuchungen, weisen einen engen inneren Zusammenhang auf. Es handelt sich dabei um sprachwissenschaftliche Phänomene, die sehr viele Verbindungen aufweisen, und dadurch gleichzeitig viele verschiedene Fragestellungen neu aufwerfen, die oft offen sind. Im Zentrum der Beobachtungen stehen dann die Fragen des Sprachgebrauches. Die Sprache ist nämlich das grundlegendste Kommunikationsmittel, welches die Menschheit als reales kulturelles Erbe betreut. Die Sprache dient gleichzeitig als Mittel der grenzüberschreitenden Verständigung, wobei als Grenzen unterschiedliche Merkmale zu verstehen sind. Am Leben einer Sprache ist also sehr markant zu beobachten, wie sich das entsprechende Land und seine Menschen entwickeln. Dies gilt natürlich auch umgekehrt, und man kann anhand der vielen Attribute eines Landes feststellen, wie intelligent und „kulturfähig“ die Menschen sind, weil die Sprache nicht nur zur Äußerung, sondern auch zur Bewusstmachung der Emotionen und inneren Gefühle genutzt wird. Auf jeden Fall bleibt die Sprache an sich ein grenzüberschreitendes Verständigungsmittel, und für die Vielfalt der Nationen und deren Sprache bleibt es weiterhin ein Kulturphänomen, mit dem man sich immer wieder auseinanderzusetzten versuchen sollte. Sprache ist nämlich trotz ihrer mannigfaltigen Eigenschaften ein flexibles System, das sich mit der Entwicklung der Menschheit und ihrer verschiedenen Gesellschaftskreisen verändert und wandelt. In dem vorliegenden Beitrag wird zunächst auf die Problematik der Transkulturalität generell in Bezug auf die Vielfalt und Mannigfaltigkeit nebeneinander existierender Nationen und ihrer kulturellen Eigenarten 
eingegangen. Es wird die Auslegung des Sprachnorm-Begriffs basierend auf Nekula, Gloy bzw. Keller verfolgt. Anschließend wird der Begriff der gegenwärtigen Mehrsprachigkeit aufgegriffen, wobei die soziolinguistische Perspektive kurz behandelt wird. Im nächsten Kapitel werden die Schwerpunkte und der Prozess der Translation erörtert. Abschließend wird die empirische Phase anhand der herangezogenen Beispiele aus der Zeitung dargestellt.

\section{Transkulturalität als Ansatzpunkt für moderne Sprachgesellschaft}

Der Begriff Transkulturalität nimmt Bezug auf eine transkulturelle Gesellschaft, wo es zur Begegnung zweier oder mehrerer unterschiedlicher oder sogar gegensätzlicher Kulturen kommt. Diese Kulturen weisen ihre Spezifika in den verschiedensten Bereichen auf, die gleichzeitig zu ausschlaggebenden Kriterien für die Unterscheidung oder Beschreibung jeder einzelnen Kultur werden. Das Leben einer Gesellschaft wirkt sich natürlich auch in der Kommunikation aus, d. h. in der sprachlichen Äußerung. Jede Sprache gehört so wie viele andere Qualitäten zum kulturellen Erbe eines Volkes. Spricht man dann von dem Phänomen Transkulturalität, muss man den sprachlichen Einfluss der Gesellschaften untereinander erwähnen. Von der sogenannten „,interkulturellen Kommunikation“ sprechen Nekula und König in ihren Beobachtungen zum Verhältnis von kontrastiver Linguistik und Sprachtypologie. Sprachliche Strukturen, die zwischen zwei unterschiedlichen Sprachen erforscht werden, werden meistens kontrastiv betrachtet. In einem solchem Bereich werden sprachliche Normen und Kontexte verglichen. Es werden verschiedene Sprachsysteme verglichen, indem man sich mit Kommunikationsnormen und Kommunikationsverhaltensweisen beschäftigt, die bei interkulturellen Begegnungen zu beobachten sind. [1]

Im Rahmen soziolinguistischer Überlegungen sollte man die Norm als Merkmal des Sprachverhaltens nicht vergessen. Der Begriff Norm ist, ,zu verstehen im Sinne einer Erwartungshaltung gegenüber bestimmten Formen des Sprachverhaltens, die in einer gegebenen Kommunikationssituation bzw. gegenüber einem bestimmten Gesprächspartner [...] als angemessen gelten." [2: 309]

Die moderne Sprachwissenschaft lehnt sich im Falle des Sprachnormbegriffs an den Lewisschen Konventionsbegriff an, der folgendermaßen erläutert wird (vgl. auch [3], [4], [5]):

"Eine Lewissche Konvention ist, sehr vereinfacht gesagt, eine
Verhaltensregularität in einer Gruppe, der jedes Mitglied folgt, jeder von jedem
erwartet, daß er ihr folgt, und der Grund jedes einzelnen, warum er genau dieser
Verhaltensregularität folgt, der ist, daß er annimmt, daß die andern es ebenfalls
tun. (Andernfalls wäre ihm eine andere Verhaltensregularität genauso lieb.)
Bezüglich dieser Erwartung muß kollektives Wissen bestehen, $d$. h. jeder muß dies
wissen und wissen, daß dies jeder weiß." [6: 136]

Sprachnormen verhelfen zur Verbesserung einer Kommunikation. Wenn man sich also nach jeweils gültigen Sprachnormen richtet, braucht man sich keine Gedanken über die Verhaltenserwartungen der Gesprächspartner zu machen. Durch die Übertragung der sprachlichen Normen über die Landesgrenze, d. h. durch sog. Zusammentreffen oder Zusammenleben zweier Sprachkulturen kommt es jedoch zu einer weiteren Herausbildung der sprachlichen Normen, die eine neue Dimension aufweisen, und zwar meistens nur innerhalb einer bestimmten sozialen Gruppe. Das menschliche Handeln bedingt bestimmte, zugleich zahlreiche gesellschaftliche Lebensbereiche, Situationen, soziale Rollen oder spezifische Kommunikationsziele, ohne Landes- bzw. Sprachgrenzen in Frage zu stellen. Deswegen muss beachtet werden, dass, wenn man eine andere Kultur leben darf, Verantwortungsanforderungen an jeden einzelnen Sprachbenutzer vorausgesetzt werden, und 
zwar die Verantwortung gegenüber dem sprachlichen Erbe. Schon Gloy hat sich dazu in seiner Normbeschreibung geäußert:

“Unter 'Sprachnormen' verstehe ich jene Teilmenge sozialer Normen [...], die durch Werturteile, Aufforderungen und (geäußerte) normative Erwartungen den Umfang der zulässigen sprachlichen Mittel, und - in Abhängigkeit von Faktoren der "Situation" sprachlichen Handels - eine spezifische Auswahl dieser Mittel bestimmen, vorschreiben oder auch nur empfehlen. 'Sprachnormen' sind also nicht etwa sprachlich fixierte Normen überhaupt, $d$. $h$. Normformulierungen, sondern soziale Normen, die - gleichgültig, ob formuliert oder nicht formuliert den Umfang, die Auswahl, den spezifischen Gebrauch von Sprachmitteln in irgendeiner Hinsicht als verbindlich festlegen" [7: 61].

Das Einhalten der Sprachnormen hängt also von vielen Faktoren ab, unter denen sowohl innenmenschliche als auch äußere Einflüsse eine Rolle spielen. Bei einer transsprachlichen Begegnung kann es leicht zum Fehlverhalten kommen, das in der Folge unzähliche Mißverständnisse im dann folgenden Handeln verursachen kann. So sind menschliche Werte wie Bereitschaft zur Annahme neuer Tatsachen und ein bestimmtes Vorwissen unentbehrlich.

Nicht zuletzt sind bei grenzüberschreitender Zusammenarbeit außersprachliche Parameter von Bedeutung. Sprache und Gesellschaft hängen aus soziolinguistischer Sicht sehr eng miteinander zusammen. Die Sprache weißt nach Linke, Nussbaumer und Portmann nicht nur Merkmale der Uniformitätsmöglichkeiten, sie reflektiert auch soziale Unterschiede und Ungleichheiten, d. h. sie wird zum Spiegel sozialer Verhältnisse. Gleichzeitig wird davon ausgegangen, dass die Sprache soziale Realität erzeugt und diese stabilisiert. [2: 311]

Außersprachliche Parameter wie unterschiedliche soziale Schichten, das Alter, das Geschlecht, soziale Rolle bzw. Situation tragen dazu bei, wie sich eine Kultur sprachlich präsentiert. Gerade die Situation ist „unter soziologischem Gesichtspunkt die Schnittstelle verschiedener relevanter aussersprachlicher Parameter. Sprachliches Verhalten in einer bestimmten konkreten Situation ist deshalb immer das Ergebnis komplexer Gewichtungs- und Evaluationsprozesse, die der einzelne Interaktant im Zusammenspiel mit den vorhandenen Kommunikationspartnern bewältigen muss, um sich schliesslich für ein angemessenes Sprachverhalten zu entscheiden." [2: 315]

\section{Gegenwärtige Vielsprachigkeit}

Vielsprachigkeit steht als Synonym für Mehrsprachigkeit. Der Begriff bezieht sich sowohl auf einen einzelnen Sprachproduzenten als auch auf die ganze Gesellschaft als Einheit und zwar nicht nur im Rahmen eines Landes. Es gibt also viele verschiedene Auffassungen, die uns erlauben, dieses Thema näher zu betrachten oder faktisch aufzugreifen.

Mehrsprachigkeit wird als ,einerseits die Fähigkeit eines Menschen, mehr als eine Sprache zu sprechen“ bezeichnet, ,andererseits versteht man unter diesem Begriff die Geltung oder verbreitete Anwendung mehrerer Sprachen in einer Gesellschaft, einem Sprachgebiet oder einem Staat."[8] Die Online-Version des Dudens spricht im Zusammenhang mit der Mehrsprachigkeit nur über „die Fähigkeit, mehrere Sprachen zu sprechen.“ [9]

Als Synonym zum Begriff Mehrsprachigkeit wird im Duden jedoch der Begriff Polylingualismus behandelt. [10] Eine andere Bezeichnung wäre dann Multilingualismus, und im Zusammenhang mit diesem Begriff wird Zweisprachigkeit bzw. Bilingualismus verwendet. [2]. Laut von Bargen wird dieser als eine wahlweise Verwendung von zwei oder mehr Sprachen durch eine Person charakterisiert. Die Ursachen der Zweit- bzw. 
Mehrsprachigkeit sind verschieden, oft sind das persönliche Gründe, es können jedoch auch soziale, sozioökonomische oder sogar politische Gründe sein. [11]

Man kann sich die Frage stellen, ob Vielsprachigkeit, Mehrsprachigkeit bzw. die Zweitsprache einen konkreten Zweck in der Gesellschaft erfüllen. Heutzutage ist Zwei- oder Mehrsprachigkeit schon ein natürlicher Bestandteil der zwischenmenschlicher Kommunikation, weil die heutige globalisierte Welt auf nebeneinander existierenden soziokulturellen Tatsachen und Existenzen beruht.

Es wurden auch verschiedene Arten der Zweisprachigkeit beschrieben und viele Studien und Forschungen auf dem Gebiet des kindlichen Spracherwerbs durchgeführt, siehe z. B. die Sprachpyramide nach Wendlandt. [11]

In der Geschichte wurde jedoch Mehrsprachigkeit nicht immer positiv gesehen. Im 20. Jahrhundert gab es viele europäische Länder, die anfingen, eine Zuwanderungspolitik zu betreiben (vor allem Deutschland, als man nach dem Zweiten Weltkrieg Arbeitskräfte im Ausland anwarb). Im Zuge dieser Politik kamen dann viele Migranten ins Land, die ihre eigene Muttersprache mit sich brachten. Damit mussten sich jedoch vor allem diejenigen Länder auseinandersetzen, die die Fremden und Ausländer aufgenommen haben. Im Rahmen des aktiven Bilingualismus, der in eine breite Gesellschaft mit einbezogen wird, ist es wichtig, das kulturelle Niveau der Sprache zu bewältigen, man soll sich bemühen zu verstehen und verstanden zu werden. [12]

Vielsprachigkeit als existierendes Phänomen, das verschiedene Gründe hat, könnte im Allgemeinen bei translatorischen Vorgängen behilflich sein. D. h., man könnte das Sprachwissen von zwei- und mehrsprachigen Personen für die Translation nutzen, da diese mehr oder weniger natürlichen MehrmuttersprachlerInnen automatisch mehrere Sprachvariablen sprechen können. Wichtig bei jeder Sprachgemeinschaft ist jedoch das sprachliche Verhalten der SprecherInnen. Damit hängt nämlich die Art des gesprochenen Codes eng zusammen. Verschiedene sozioökonomische Verhältnisse schaffen unterschiedliche soziale Schichten, die explizit durch unterschiedliches Sprachverhalten gekennzeichnet sind. [2: 297]. So stellt sich gleichzeitig die Frage, ob Mehrsprachigkeit, die heutzutage immer positiver angesehen wird, die Sprachbarrieren abbaut, oder ob sie vielleicht auch neue noch nicht bekannte Grenzen schaffen kann. Jede Sprache, vielleicht sogar auch eine neu enstandene gesprochene Variante, die als Mischung von zwei Sprachen entstanden ist, bedient sich eigener Regeln, die an sich keine negativen Auswirkungen innerhalb dieses Soziolektes verursachen können, die jedoch für die außerhalb Stehenden ein Problem bei translatorischen Prozessen darstellen könnten. Mit der Defizittheorie beschäftigen sich Linke, Nussbaumer und Portmann im Studienbuch Linguistik im Rahmen der Differenztheorie, wo die Sprachunterschiede nicht mehr als Mangel interpretiert werden, sondern als Andersartikgeit. [2:199]

Schlieben-Lange unterscheidet multilinguale Kompetenz bei einem einzelnen Sprecher. Sie spricht von zwei verschiedenen Aspekten der Heterogenität der Sprecherkompetenz, nämlich die sog. aktive und passive multilektale Kompetenz. Im Rahmen der aktiven multilektalen Kompetenz erwähnt sie die Verfügbarkeit über mehrere Varianten der Muttersprache des Sprechers, zwischen denen er umschalten kann. Dieses Umschalten geschieht in Abhängigkeit von der Situation, dem Auditorium, dem Thema usw. Weiter kann es sich zwischen regionalen Varietäten, Soziolekten und stilistischen Varietäten bzw. deren Überschneidungen abspielen. Das Umschalten bzw. code-switching erlaubt solchen SprecherInnen „in eine andere Varietät zu ,übersetzen“, wobei sie Gefahr laufen, ihre Adaptionsregel überzugeneralisieren und Hyperkorrektismen zu produzieren.“ [13: 95-96] 
Die passive multilektale Kompetenz steht nach Schlieben-Lange im Zusammenhang mit der Existenz einer gewissen Menge an passiven Kenntnissen über andere Subsysteme der Sprache. Damit ist gemeint, dass man sich z. B. bei längeren Aufenthalten in anderen Dialektgebieten Fähigkeiten im Decodieren und Umsetzen in die eigene Sprachform aneignen kann, ohne dass man die Fähigkeit hätte, den anderen Dialekt selbst zu sprechen. [13: 96] Als Beispiel für ein gewisses Verstehen von Wörtern mit denen man in Berührung kommt, diese versteht, aber selber nicht benutzt, ist z. B. der Bereich der Massenmedien. Es stellt sich hier die Frage, ob die Andersartikgeit als andersartige semantische Kompetenz vieler Sprachbenutzer allgemein positiv betrachtet werde sollte, oder ob sie im Rahmen der Sprachbarrierenproblematik intensiver wahrgenommen werden soll.

Im Rahmen der Mehrsprachigkeit und aus der Perspektive, wie dieser Begriff zu verstehen ist, gibt es Phänomene, die die Mehrsprachigkeit einbeziehen oder sie von den anderen Phänomenen abgrenzen. Dies betrifft z. B. die Bezeichnung der Diglossie, die in der deutschsprachigen Schweiz bekannt ist. Bei Diglossie geht es um „1. eine Form der Zweispsrachigkeit, bei der die eine Sprachform die Standardsprache darstellt, während die andere im täglichen Gebrauch, in informellen Texten verwendet wird, 2. Vorkommen von zwei Sprachen in einem bestimmten Gebiet (z. B. Englisch und Französisch in Kanada), 3. das Auftreten von zwei ausgebildeten Varianten der Schriftsprache in einem Land (z. B. Bokmal und Nynorsk in Norwegen)“. [14]

Eine andere Art der Mehrsprachigkeit stellt z. B. die Benutzung von Entlehnungen aus anderen Sprachen oder das sogenannte Code-Switching und Code-Mixing dar. Die letzen zwei Phänomene beruhen auf bewusstem bzw. unbewusstem Ablösen zweier oder mehrerer Sprachen innerhalb eines Sprechaktes. Bei Code-Mixing fehlt eine klar abgegrenzte Sprache der Interaktion. Wenn also nicht klar ist, welche der zwei Sprachen die Matrixsprache ist, spricht man von Code-Mixing. Auf dem Prinzip eines intensiven Sprachkontaktes und aufgrund der gegenseitigen sprachlichen Beeinflussung entstehen sog. Pidgin- und Kreolsprachen, bei denen eine nicht regulierbare Mischung der Sprachen den Wesenszug darstellt. Nach Schwitalla hat in diesem Fall der Sprachwechsel oft keine semantische oder diskurssteuernde Funktion mehr. [15: 55]

Bei dem schon oben erwähnten Code-Switching geht es um einen Sprachwechsel bzw. um das Umschalten von einer Sprache bzw. Variante in eine andere. Für Mehrsprachigkeit bzw. ihr folgendes Code-Switching gibt es verschiedene Gründe, und daher unterscheidet man auch verschiedene Typen der Mehrsprachigkeit. Laut Riehl gibt es:

a) individuelle Mehrsprachigkeit, die sich auf den einzelnen Sprecher bezieht,

b) territoriale Mehrsprachigkeit, bei der der Sprachgebrauch in mehrsprachigen Staaten oder Regionen manifestiert wird,

c) institutionelle Mehrsprachigkeit, bei der es sich um die Verwendung mehrerer Arbeitssprachen in Institutionen handelt. [16] Andere Sprachforscher zählen zu dieser Aufteilung auch noch die sogenannte gesellschaftliche Mehrsprachigkeit hinzu, bei der ein gegenseitiges Durchdringen der Sprachgemeinschaften herrscht, wie in Überlappungsgebieten an Sprachgrenzen. [17]

Die Ursachen der Mehrsprachigkeit sind verschieden, generell beruhen sie jedoch meistens auf politischen Gründen (Kolonialisierung, Globalisierung), auf sozioökonomischen Gründen (Migration) und auf persönlichen Gründen (Bilingualismus = natürliche Mehrsprachigkeit, Eigenfremdsprachenerwerb $=$ schulische bzw. künstliche Mehrsprachigkeit).

Was die Form der Mehrsprachigkeit angeht, kann man, so wie es gesprochene und geschriebene Formen der Sprache gibt, auch diese zwei Qualitäten der Betrachtung auf 
diesem Gebiet erfassen. In der Praxis stößt man natürlich viel öfter auf die gesprochene Variante der Mehrsprachigkeit, jedenfalls was die individuelle und territoriale Mehrsprachigkeit angeht, handelt es sich hier um die eher natürliche Angeignung der Zweitsprache. Roche spricht von Mehrsprachigkeit, wenn ,ein Sprecher sich tatsächlich ähnlich flüssig und problemlos in wesentlichen Bereichen mehrerer Sprachen ausdrücken kann.“ [18: 80] Daraus ergibt sich die These, die Roche ebenso unterstützt, dass es viele Zwischenstufen der Mehrsprachigkeit gibt, die allerdings auf diversen Grundlagen beruhen. In Bezug auf die translatorischen Bemühungen aller Art wäre eine ausgeglichene Mehrsprachigkeit von Vorteil, denn diese Form der Mehrsprachigkeit zielt nicht nur auf die kognitive Entwicklung jedes einzelnen Sprechers, sondern auch auf die äußerst positive Bereicherung der Translationswissenschaft an sich.

\section{$3 \quad$ Translation als transkultureller Prozess}

Die Translation ist ein Begriff, der über die Grenzen hinaus zu verstehen ist. Er geht auf das Lateinische zurück, dem Duden Universalwörterbuch nach heißt Translation „[1: lat. translatio $=$ das Versetzten, die Übersetzung, zu: translatum, 2: Part. von transferre = hinüberbringen, 3: frz. translation < lat. translatio]: 1. (Fachspr.; bildungsspr.) Übertragung, Übersetzung [...]“. [19: 1596] Der Inhalt dieser Bezeichnung hängt sehr eng mit der menschlichen Verständigung unter dem Einfluss verschiedener Sprachen zusammen. Um sich über die Grenzen einer Nation verständigen zu können, braucht man nämlich die Translation als Prozess allmählicher Übertragung einzelner Teile eines Sprachsystems in ein anderes. Als Teile eines Sprachsystems sind viele Bestandteile existierender sprachlicher Ebenen zu bestimmen, d. h. Wörter und Lexeme des Gesamtwortschatzes einer Sprache, konkrete Sprechakte, die im Rahmen eines Diskurses entstehen können, morphologische und syntaktische Gegebenheiten der konkreten sprachwissenschaftlichen Ebenen der Linguistik u. a.

Wenn man die Begriffe Übersetzung und Übertragung genauer betrachtet, wird man feststellen, dass sie im weiteren Sinne des Wortes einander gleichen, im engeren Sinne des Wortes jedoch einige Unterschiede aufweisen. Bei einer Übersetzung handelt es sich um das Übersetzen von sprachlicher Zeichen von einer Sprache in die andere. Eine Übertragung ist ein eher breiterer Begriff, man kann allgemein Daten aller Art übertragen, wobei man bei Daten auch sprachliche Zeichen einbeziehen kann. Bei einem Sprachkontakt geht es jedoch auch um Übertragung von solchen sprachlichen Elementen, die unter soziolinguistischen Aspekten untersucht werden können. „Soziolinguistische Fragestellungen fokussieren besonders das Verhalten von Sprechern in bestimmten Domänen, [...]“. [20: 46]

Der Begriff „Translation“ wird allerdings eher mit dem simultanen Dolmetschen verbunden. Das Verb „dolmetschen“ wird charakterisiert als: „a) einen gesprochenen od. geschriebenen Text für jmdn. mündlich übersetzen [...]“. [19: 389]

Mit Translation hat man es ständig zu tun, auch wenn man sich gerade nicht wissenschaftlich mit dieser Disziplin beschäftigt. Soziologisch gesehen könnte man die Gesellschaft teilen in eine Gruppe, die sich bewusst mit Translation beschäftigt, und in den anderen Teil, der sich mit einzelnen Merkmalen bzw. Teilprozessen der Translationswissenschaft auseinandersetzt und zwar ganz unbewusst, ohne es praktisch wahrzunehmen.

Die erst genannte Gruppe der Menschen spezialisiert sich gerade und wissentlich auf die Fertigkeit dieser sprachwissenschaftlichen Disziplin, und man könnte sagen, dass sie als höhere Kunst bezeichnet werden kann. Wie Weinitschke in ihrer Abhandlung über translatologische Probleme und Methoden referiert, kann man sich bei Translation nicht lediglich mechanisches Übersetzten vorstellen: „Translatorisches Handeln bezieht sich nicht 
einfach auf das Übersetzen auf Wort- beziehungsweise Satzebene, sondern meint die gesamte Produktion von sogenannten Botschaftsträgern (dies können Texte, aber auch außersprachliche, non-verbale Elemente sein).“ [21: 2] Daraus geht hervor, dass beim Übersetzen bzw. Dolmetschen Experten handeln müssen, von denen nicht nur sprachliche, sondern auch andere offenbare Kompetenzen verlangt werden. Die Translatoren sollten auf sie übertragene „Probleme“ erfassen und schließlich Lösungsmöglichkeiten auffinden und auswählen. Um erfolgreich zu arbeiten, müssen Experten mit dem Auftraggeber kooperieren. Kooperationsmodelle und Handlungskonzepte zu dieser Problematik stellt Holz-Mänttäri vor. [22: 348-374]

Translatoren arbeiten also in einem sehr komplexen, interkulturellen Handlungsgebilde, und müssen dazu fähig sein, nicht nur die in Auftrag gegebenen Texte und Texteinheiten als Übersetzung zu erstellen bzw. zu dolmetschen, sondern auch als gewisse Berater zu handeln. Sie entscheiden über die vermittelnden Inhalte, die entsprechende Sinnhaftigkeit des Inhaltes und über die ganze Qualität des Textes.

Mit dem Herausfinden und der folgenden sprachlichen Äußerung der außersprachlichen bzw. non-verbalen Elemente einer Texteinheit muss vorsichtig vorgegangen werden. Die Translatoren können auf Kulturbarrieren stoßen, und es kann in der Zielkultur auf Unverständnis oder sogar Ablehnung treffen.

Mit der Rolle der Übersetzer und Dolmetscher in der Gesellschaft beschäftigt sich auch Muschner in ihrer Betrachtung der Grenzen des Übersetzbaren. Sie zielt auf die Personalität und Professionalität des einzelnen Übersetzers ab: „Übersetzen basiert folglich auf einem tiefen Respekt dem Verfasser des Ausgangstextes gegenüber, dessen Inhalte, Übersetzungen, Emotionen und Haltungen der Übersetzer zunächst erst einmal selbst verstehen muss, ehe er sie in der anderen Sprache darstellen kann.“ [23: 423]

Im engeren Sinne des Wortes kann Translation auf drei Grundebenen realisiert werden: auf der phonischen, auf der graphischen und auf der gestischen Ebene der Sprache. Die Sprachverwendung verläuft meistens auf Grund der Produktion hörbarer Laute bzw. schriftlicher Zeichen, und die Sprache wird medial als phonisch oder graphisch realisiert. Das Sprechen überwiegt über anderen Erscheinungsformen der Sprache. [15: 19]. Man kann also Translation im Sinne einer mündlichen Produktion mit der schriftlichen Produktion nicht vergleichen. Das Dolmetschen eines mündlichen Sprechaktes und das Übersetzen eines schriftlichen Textes weisen ihre eindeutigen Merkmale auf, und man müsste sich mit deren Problematik separat beschäftigen. Darüber hinaus wird Translation durch eine der inneren Qualitäten ihres Bereiches, und zwar durch die richtige Wahrnehmung der zum Ausdruck gebrachten Realität gekennzeichnet. Dies hängt natürlich sehr eng mit der Dolmetscher- bzw. Übersetzerpersönlichkeit und ihren translatorischen Künsten zusammen. Es gibt also viele verschiedene Faktoren, die im Bereich Translation eine immense Rolle spielen, und die bei jedem translatorischen Akt beachtet werden sollten.

\section{$4 \quad$ Praktische Anwendung der Transkulturalität und Mehrsprachigkeit am Beispiel der Sprache der Zeitung}

\subsection{Zielsetzung und methodisches Verfahren}

Der Gegenstand der empirischen Betrachtung ist festzustellen, in wie weit und welche fremdsprachlichen Ausdrucksmittel in der Zeitungssprache vorkommen. Das ist allerdings sehr gut an den modernsten Kommunikationsmedien $\mathrm{zu}$ beobachten, die sonst als Informationsvermittler dienen. Eine der wichtigsten Funktionen der Medien allgemein ist, die Kunden auf dem neuesten Stand zu halten, d. h. aktuell zu sein. Die persuasive Funktion der 
Zeitungssprache wird jedoch auch durch die Nutzung des fremden Wortguts aufgefasst. Deshalb ergab sich die Frage nach der Häufigkeit der Entlehnungen, weiterhin auch nach der qualitativen Besetzung der Stelle durch ein Fremd- bzw. Lehnwort.

Aufgrund der qualitativen und quantitativen Analyse soll bestätigt werden, dass das Englische die am häufigsten vorkommende Gebersprache ist, und die Sprachkultur der Schlagzeilen in zahlreichen Fällen auf der alten Lingua franca, nämlich auf dem Lateinischen, beruht.

Transkulturalität und Mehrsprachigkeit gehen Hand in Hand durch ihre Existenz. Sie ergänzen sich oder bedingen sogar einander. Sehr oft kann man deshalb beiden gerade im Bereich der Medien begegnen. Obwohl sich neue Medien wie Internet und auf dessen Basis existierende Chat-Plattformen in den Vordergrund drängen und sich einer nennenswerten Beliebtheit erfreuen, stehen Printmedien nicht weit hinter diesen. Die Zeitungssprache kann sowohl aus der soziolinguistischen Perspektive als auch aus der Sicht der reinen Linguistik betrachtet werden, deswegen eignet sie sich gut für eine nähere Untersuchung, wo soziale Faktoren und rein sprachwissenschaftliche Aspekte sich berühren. Als Probe für den vorliegenden Beitrag wurde ein Korpus zusammengestellt, das Mehrsprachigkeit und die Anwesenheit mehrerer Kulturen über die Grenzen hinaus bestätigen kann. Das Korpus wurde manuell erstellt. Zur Verfügung stand die Sprache des Internetmediums Spiegel Online. [24] Der Zeitraum wurde auf den Monat April des Jahres 2017 begrenzt. Als Gegenstand der Beobachtung wurde die Lexik der Schlagzeilen dieses Online-Magazines gewählt. Die Untersuchung wurde auf das fremdsprachliche Lexikum ausgerichtet, sowie das Vorkommen anderer Sprachen im Rahmen der sonst auf Deutsch gebildeten Schagzeilen. Die Herkunft einzelner fremdwörtlicher Ausdrücke wurde mit Hilfe der Online-Quelle Duden [25] und dem Digitalen Wörterbuch der deutschen Sprache [26] überprüft.

\subsection{Empirische Feststellungen}

Das Eindringen fremdsprachiger Elemente zeigte sich an dem untersuchten Korpus auf mehreren Ebenen der Vorgehensweise. Zuerst wird die lexikalische Ebene der fremden Sprachen allgemein betrachtet, dann wird auch über einzelne Gebersprachen referiert. Die quantitative Untersuchung hat gezeigt, dass Anglizismen, vom Umfang her, die meistvorkommende Gebersprache ist. In der gesamten Menge der zweitausenddreihundertneunundachzig Schlagzeilen wurden in einhundertsechzehn Schlagzeilen einhundertvierzig Anglizismen gefunden. Den zweiten Platz nehmen Latinismen ein, es wurden in vierundsiebzig Schlagzeilen sechsundsechzig Latinismen gefunden. Den dritten Platz nehmen Gallizismen mit achtundvierzig Wörtern französischer Herkunft in einundsechzig Schlagzeilen ein. Auf dem vierten Platz findet man Gräzismen mit neunzehn Wörtern griechischer Herkunft in zweiundzwanzig Schlagzeilen. Danach folgen Italianismen mit fünfzehn Wörtern italienischer Herkunft in vierzehn Schlagzeilen. Den sechsten Platz belegen Hispanismen mit vier Ausdrücken spanischer Herkunft in drei Schlagzeilen. Den Rest übernehmen drei Gebersprachen, das Arabische mit zwei Ausdrücken (Hummus und Razzia), das Irische (Boykott) und das Russische (Ikone).

Was die qualitative Analyse angeht, wäre es logisch zu supponieren, dass das Englische heutzutage ganz offensichtlich alle Sphären der menschlichen Tätigkeit durchdringt und auch bewusst auf den Bereich der Medien zielt. Als Anglizismen erweisen sich vorrangig Substantive. Die folgenden Anglizismen sind diejenigen, die zumeist und oft auch mehrmals verwendet wurden: Newsblog, Fake News, Deal, Streaming, Quiz, In, Boom u. a. Sehr oft kommen Wortverbindungen vor, die mehrere fremde Elemente beinhalten, d. h. dass es in erster Linie die Wortbildungsart Komposition bzw. Hybridbildung betrifft. Es kommen Komposita vor, die auf zwei bzw. mehreren Sprache beruhen, z. B.: Nahrungsmitteldesign, Outdoormarket, Faktencheck, Lauf-Quiz, Teambus, Serien-Update. Nicht selten waren es 
auch solche Schlagzeilen, die ganz auf Englisch bzw. ganz fremdsprachlich erschienen sind, z. B.: Carpools, Jobtickets (4. 4. 2017), Great-Barriere-Reef (10. 4. 2017), Trump-Boom (26. 4. 2017), Promi-Date (10. 4. 2017), Dubiose Onlineshops (13. 4. 2017). Natürlicherweise beinhalten viele der ganz fremdsprachlichen Schlagzeilen die Merkmale der Anpassung des fremden Lexikums an das deutsche Sprachsystem, indem man 1. bei den Substantiven die Großschreibung der Anfangsbuchstaben einhält, 2. die Flexion inklusive der Steigerung beachtet. Dies ist speziell an Adjektiven und Verben markant: Dialog mit einem Kleinkind: „Ich bin die coolste Person im Universum!“ (18. 4. 2017), Windows 10: Warum Microsoft nicht will, dass Sie manuell updaten? (27. 4. 2017), Katy Perry googelt sich selbst. (15. 4. 2017)

Es ist interessant, dass viele Wörter lateinischer Herkunft oder Ausdrücke, die auf dem Lateinischen basieren, wie das Französische oder das Spanische, zu einer solchen Art der Entlehnungen gehören, die schon eine Zeit lang im deutschen Sprachsystem existieren. Sie können, pragmatisch gesehen, so aufgenommen werden, als ob sie schon längst in das deutsche Sprachsystem integriert worden wären. Man verwendet diese sowohl in der gesprochenen als auch in der geschriebenen Sprache fast schon automatisch, und empfindet sie nicht mehr als fremde Lexik. Im untersuchten Korpus tauchten folgende Latinismen auf, z. B.: Terror, Ex-, Ritual, Korruption, versus, Prozess, Propaganda, Index, Defekt, Solar, extra, Tribune, Konjunktur, Zensur, Suizid, Sanktion u. a. Wie daraus erkennbar ist, handelt es sich vorwiegend um substantivische Lexeme, von den anderen Wortarten findet man eher wenige. Das bedeutet, dass hier hauptsächlich die Benennungsfunktion ihre Relevanz findet, was auch der Fall der Sprachen ist, die von dem Lateinischen ausgehen. Bei den Gallizismen waren es folgende, die am häufigsten vertreten waren: Affäre, Debatte, Debakel, Eklat, Manöver, Spektakel, Remis, Attacke, Bankier, Branche, Desaster, Visier, Recherche, Appell, Engagement, Allianz, Reportage, Saison, Tour, attraktiv. Nicht uninteressant ist das Vorkommen der Gräzismen, von denen der Großteil auch nicht mehr als fremde Lexik empfunden wird, sondern diese aufgrund der Übertragung der Sprachkultur vor allem aus den fachlich ausgerichteten Bereichen wie Medizin oder Rechtswesen eher als integriert und heimisch aufgefasst wird. Es geht um Konfixe: Bio-, Neo-, Mega-, Anti-, Pharma-, oder Ausdrücke wie Skandal, Prognose, Narkose, Diagnose, Therapie, Taktik, Hysterie. Von den Italianismen erschienen im untersuchten Korpus: Alarm, Co., andiamo, Biennale, Grazie, mille, Scenario. Die Hispanismen vertreten: Liga, primera, el und clásico. Es kommen auch solche Komposita vor, die rein auf Latinismen, Gallizismen, Gräzismen und deren Kombination basieren, und die man auch als zusammengesetzte Komplexe wahrnimmt. Die Beispiele dafür sind z. B.: Terrorprozess, Terrorpropaganda, Immobilien-Index, Info-Index, Koalitionsoption, Korruptionsskandal, Korruptionsaffäre, Konjunkturprognose, Pharmabranche, Biobranche, Biosphäre.

Sehr interessant und als eine passende Art der Schlagzeilenbildung zeigt sich die Verbindung einzelner Events mit bekannten Namen bzw. Markennamen. Im bearbeiteten Korpus wurde jede Menge solcher Wortverbindungen gefunden, z. B.: Brexit-Folgen, Sharapova-Comeback, Ex-Siemens-Chef, Fake-News-Debatte, Anti-Populismus-Buch u. a.

Beliebt bei den JournalistInnen sind oft auch verschiedene Hybridbildungen bzw. Komposita, die auf der Wortbildungsart Kontamination beruhen. Auf jeden Fall dient das Verwenden der außerordentlichen Ausdrücke, aus der fremden Lexik, vorwiegend aus der englischen Sprache, dazu, die Leserschaft zum Lesen zu motivieren. Ein gegenwärtig oft vorkommendes Phänomen, das auch innerhalb der geschriebenen Form der Sprache zu finden ist, ist der Wortwechsel bzw. das Code-Switching. Da der Wortwechsel in beiden Grundaussageformen der Sprache seine Regeln hat, nach denen er verläuft, findet man in der Sprache der Medien nicht selten Code-Switching-Fälle, bei denen gern von einer Sprache in eine andere 
gewechselt wird. Dieser Prozess hat zahlreiche Ursachen, und in der journalistischen Branche kann man dieses Verfahren als eher bewusst und gezielt betrachten.

\section{Fazit}

Da es seit dem Beginn der Menschheit verschiedene Gesellschaftsstrukturen und Völker gibt, ist es selbstverständlich, dass es beim gegenseitigen Kontakt zu einer gewissen Mischung der Kulturen und verschiedener kultureller Elemente kommt. Deshalb darf man die Begegnung der Kulturen nicht etwa als einen neuen, mit der Globalisierung erscheinenden Prozess betrachten. Es tauchen vielleicht neue Bezeichnungen für alte Erscheinungen auf, die menschliche Begegnung im Rahmen ihrer Kulturen fand jedoch schon immer statt. Der Unterschied zwischen damals und heute besteht jedoch in einer unterschiedlichen Art der Wahrnehmung der zwischenmenschlichen und interkulturellen Begegnung. Seit den letzten Jahrhunderten gibt es hinreichende und immer weitere Beweise dafür, wie gut bzw. nicht so gut einzelne Kulturen miteinander ausgekommen sind. Sprache, die als Grundlage für eine erfolgreiche Verständigung steht, gehört an sich zum kulturellen Erbe jedes einzelnen Volkes. Das Niveau der sprachlichen Kommunikation stellt einen der wichtigsten Ansatzpunkte dafür dar, wie erfolgreich man im transkulturellen Dialog sein kann. Dafür sind auch Medien und ihre Sprache ein Beweis, um so mehr als in diesem Bereich Interkulturalität im Rahmen der Mediensprache stark spürbar ist. Wie aus der praktisch ausgerichteten Untersuchung hervorgeht, bedient sich die Zeitungssprache sehr gern der fremden Lexik, d. h. der Sprachen anderer Nationen und Kulturen. Das Ergebnis der sprachwissenschaftlichen Analyse zeigt, dass die größte Gebersprache heutzutage das Englische ist, gefolgt von dem Lateinischen, Französischen und Griechischen. Es ist interessant zu beobachten, dass eine breite Auswahl an der Lexik nicht durch die eine Sprache begrenzt ist, womit sie ständig neue und attraktive Ansätze und Impulse vermittelt. Durch die fremdsprachliche Lexik werden nicht nur nahe oder weit entfernte Kulturen repräsentiert, sondern in gewissen Situationen auch die Denkweisen und Ansichten auf die gegebene Realität. Obwohl das Englische sehr rasch ins deutsche Sprachsystem eindringt, bildet der Anglizismenanteil im Korpus der Schlagzeilen mit einer oder mehreren fremdsprachlichen Komponenten nur $12 \%$ der gesammelten Entlehnungsbeispiele. Wenn man die innersprachlichen Aspekte des Schlagzeilenkorpus betrachtet, wird die Beliebtheit der Komposita bestätigt. Diese liegt bei $60 \%$ des fremdwörtlichen Wortbestandes. Einbezogen wurden Komposita mit einem bzw. mehreren fremdsprachlichen Elementen in der Schlagzeile.

Um eine Schlussfolgerung zu ziehen, kann gesagt werden, dass der grenzüberschreitende sprachliche Einfluss die deutsche Sprache eher bereichert, als dass er ihr schaden könnte. Um mit anderen Sprachkulturen auszukommen, muss auch einiges von der herkommenden Substanz angenommen werden, obwohl jede Sprache Doppelstöckigkeiten aufweist, was jedoch im Rahmen der Zeitungssprache eher als Vorteil angenommen werden kann.

\section{Literatur}

[1] KÖNIG, E.; NEKULA, M.: Zum Verhältnis von Kontrastiver Linguistik und Typologie: Präpositionen im Vergleich. In: Nekula, M., Šichová, K., Valdrová J. (eds.): Bilingualer Sprachvergleich und Typologie: Deutsch - Tschechisch. Tübingen: Stauffenburg / Julius Groos. IDS-Reihe Deutsch im Kontrast, 28. pp. 15-46 [online]. 2013 [Abrufdatum 2016-01-18] Verfügbar unter WWW: http://www.bohemicum.de/fileadmin/Downloads/nekula/nekula_clanky/02Koenig_Nek $\underline{\text { ula.pdf }}$

[2] LiNKE, A.; NUSSBAUMER, M.; PORTMANN, P. R.: Studienbuch Linguistik. Tübingen: Max Niemeyer Verlag. 2001. ISBN 978-3-8252-152-2. 
[3] NERIUS, D.: Zur Bestimmung der sprachlichen Norm. In: STUF - Language Typology and Universals. 33(1-6), 1980, pp. 365-370. ISSN 1867-8319. DOI: $10.1524 /$ stuf.1980.33.16.365

[4] BARTSCH, R.: Sprachnormen: Theorie und Praxis. Tübingen: Max Niemeyer Verlag. 1985. ISBN 978-3-11-093587-5.

[5] RIPFEL, M.: Die normative Wirkung deskriptiver Wörterbücher. In: Hausmann F. J. et al. (eds.), Wörterbücher. Ein Internationales Handbuch zur Lexikographie. Band 1, Berlin/ New York, 1989. pp. 189-207.

[6] KELLER, R.: Zeichentheorie. Tübingen: Francke. 1995. ISBN 3-8252-1849-X.

[7] GLOY, K.: Sprachnormen I. Linguistische und soziolinguistische Analysen. Stuttgart: Fromman-Holzboog, 1975.

[8] Mehrsprachigkeit. In: Wikipedia. [encyclopedia online]. [Abrufdatum 2015-04-18] Verfügbar unter WWW: https://de.wikipedia.org/wiki/Mehrsprachigkeit

[9] Mehrsprachigkeit. In: Duden. [online]. [Abrufdatum 2015-04-18] Verfügbar unter WWW: https://www.duden.de/rechtschreibung/Mehrsprachigkeit

[10] Polylingualismus. In: Duden. [online]. [Abrufdatum 2015-04-18] Verfügbar unter WWW: https://www.duden.de/rechtschreibung/Polylingualismus

[11] Die Sprachpyramide (nach W. Wendlandt). In: Kinder-Welten entdecken. p. 47. [online]. [Abrufdatum 2018-10-30] Verfügbar unter WWW: https://www.vhsst.de/familienwelten/wpcontent/uploads/2011/12/Erzieherbroschuere_Spracherwerb.pdf

[12] SVOBODOVÁ, S.: Die Perzeption, das Verständnis und der Gebrauch der Fremdsprache, näher der deutschen Sprache. [online]. 2008 [Abrufdatum 2015-04-18] Verfügbar unter WWW: https://is.muni.cz/th/r1obl/bakalarska_prace.pdf

[13] SCHLIEBEN-LANGE, B.: Soziolinguistik. Eine Einführung. Stuttgart: Kohlhammer. 1991. ISBN 978-3170112377.

[14] Diglossie. In: Duden. [online]. [Abrufdatum 2015-06-17] Verfügbar unter WWW: http://www.duden.de/rechtschreibung/Diglossie

[15] SCHWITALLA, J.: Gesprochenes Deutsch. Eine Einführung. Berlin: Erich Schmidt Verlag GmbH. 2006. ISBN 978-3-5030-9805-7.

[16] RIEHL, C. M.: Die Bedeutung von Mehrsprachigkeit. [online]. 2006 [Abrufdatum 2015-06-16] Verfügbar unter WWW: https://www.ifm.daf.unimuenchen.de/literatur/literatur_prof_riehl/bedeutung_mehrsprachigkeit.pdf

[17] Mehrsrpachigkeit. In: Wikipedia. [encyclopedia online]. [Abrufdatum 2018-10-31] Verfügbar unter

WWW: https://de.wikipedia.org/wiki/Mehrsprachigkeit\#Gesellschaftliche_Mehrsprachigkeit

[18] ROCHE, J.: Natürliche Mehrsprachigkeit als Mittel der Integration. In: Neuland, Eva (eds.), Variation im heutigen Deutsch: Perspektiven für den Sprachunterricht. [online]. Frankfurt am Main: Peter Lang Verlag, 2006. [Abrufdatum 2015-06-16]. Verfügbar unter WWW: http://core.ac.uk/download/files/454/12174385.pdf

[19] Duden: Deutsches Universalwörterbuch. Dudenverlag: Mannheim, Leipzig, Wien, Zürich. 2003. ISBN 3-411-05505-7. 
[20] RIEHL, C. M.: Sprachkontaktforschung. Eine Einführung. 3. Auflage. Tübingen: Narr Verlag. 2014. ISBN 978-3-8233-6826-7.

[21] WEINITSCHKE, G.: Translatorisches Handeln. [online]. 2013. [Abrufdatum 2015-1128] Verfügbar unter

WWW:

http://carstensinner.de/Lehre/uebersetzungswissenschaft/dossiers2013/Dossier_Translat orisches_Handeln_Weinitschke.pdf

[22] HOLZ-MÄNTTÄRI, J.: Translatorisches Handeln - theoretisch fundierte Berufsprofile. In: Snell-Hornby (Hg.): Übersetzungswissenschaft. Eine Neuorientierung. Tübingen: Francke. 1986. ISBN 978-3-8252-1415-9.

[23] MUSCHNER, A. (2015): Grenzen des Übersetzbaren. In: Kusová J., Malechová M., Vodrážková L. (eds.), Deutsch ohne Grenzen. Linguistik. Brno: Tribun EU. 2015. pp. 421-438. ISBN 978-80-263-0939-0.

[24] Schlagzeilenbeispiele. In: Spiegel Online. [online]. [Abrufdatum 2017-04-30] Verfügbar unter WWW: http://www.spiegel.de/

[25] Duden. [online]. [Abrufdatum 2017-08-15] Verfügbar unter WWW: http://www.duden.de/suchen/dudenonline

[26] DWDS: Digitales Wörterbuch der deutschen Sprache. [online]. [Abrufdatum 2017-0828] Verfügbar unter WWW: https://www.dwds.de/

Mgr. Magdalena Malechová, Ph.D. 


\section{ÚVAHA NAD TRANSKULTURNÍ DIMENZÍ MULTILINGUALISMU \\ V SOCIOLINGVISTICKÉM KONTEXTU}

Současný fenomén transkulturality dnes představuje setkávání dvou a více kultur, které vykazují nejen společné, ale především rozdílné a protichůdné aspekty. Kultura každého národa je jedinečná, a proto skýtá řadu originálních aspektů. Kdykoli tedy dojde k setkání dvou a více kultur, dochází k prolínání rozmanitých kulturních prvků toho kterého národa. Jazyk patří $\mathrm{k}$ jednomu $\mathrm{z}$ nejdůležitějších odkazů, který nám byl našimi předky předáván jako součást kulturního dědictví. Současný moderní hospodářský a společenský vývoj, který lze pozorovat zejména $\mathrm{v}$ komunikačních médiích, nás jako uživatele jazyka nutí brát nejnovější druh médií jako výzvu, a podílet se na prezentaci kultury v rámci dnešního globalizovaného světa napříč národy a jejich kulturami.

\section{REFLECTION ON TRANS-CULTURAL DiMENSION OF MULTILINGUALISM IN SOCIOLINGUISTIC CONTEXT}

Transculturality as a current phenomenon is the encounter of two or more cultures, which show not only common but particularly different or contradictory aspects. The culture of each nation is unique and therefore has a number of specific features. Thus, when two or more cultures meet, their diverse characteristics blend together. The language belongs to one of the most important legacies that our ancestors left to us as part of our cultural heritage. The current modern economic and social development, which can be observed, for example, in the communication media, drives language users to take the latest media as a challenge and participate in the presentation of culture in today's globalized world across nations and their cultures.

\section{ROZWAŻANIA NAD TRANSKULTUROWYM WYMIAREM WIELOJĘZYCZNOŚCI W KONTEKŚCIE SOCJOLINGWISTYCZNYM}

Współczesny fenomen transkulturowości przedstawia dziś spotkanie dwóch i więcej kultur, które wykazują nie tylko wspólne, ale przede wszystkim różne i rozbieżne aspekty. Kultura każdego narodu jest niepowtarzalna i dlatego ma szereg oryginalnych aspektów. Za każdym razem, gdy dochodzi do spotkania dwóch i więcej kultur, następuje przenikanie różnorodnych elementów kulturowych danego narodu. Język należy do najważniejszych elementów spuścizny przekazanych nam przez naszych przodków jako część dziedzictwa kulturowego. Współczesny rozwój gospodarczy i społeczny, który można zaobserwować w szczególności np. w mediach komunikacyjnych, zmusza nas, jako użytkowników języka, do przyjmowania najnowszych rodzajów mediów jako wyzwania oraz do współuczestniczenia w prezentacji kultury w ramach współczesnego, zglobalizowanego świata na wskroś narodów i ich kultur. 